Tropical Journal of Pharmaceutical Research, April 2009; 8 (2): 173-179

(C) Pharmacotherapy Group, Faculty of Pharmacy, University of Benin Benin City, 300001 Nigeria.

All rights reserved.

Review Article

Available online at http://www.tjpr.org

\title{
Chemical Penetration Enhancers for Transdermal Drug Delivery Systems
}

\author{
Inayat Bashir Pathan ${ }^{1 \star}$, C Mallikarjuna Setty ${ }^{2}$ \\ ${ }^{1}$ Department of Pharmaceutics, M.E.S College of Pharmacy, Sonai. Maharashtra, ${ }^{2}$ Department of Pharmaceutics, \\ Sarasvati College of Pharmacy, Moniabad, Andhra Pradesh, Indian
}

\begin{abstract}
Skin as an important site of drug application for both local and systemic effects. However in skin, the stratum corneum is the main barrier for drug penetration. Penetration enhancement technology is a challenging development that would increase the number of drugs available for transdermal administration. The permeation of drug through skin can be enhanced by both chemical penetration enhancement and physical methods. In this review, we have discussed the chemical penetration enhancement technology for transdermal drug delivery as well as the probable mechanisms of action.
\end{abstract}

Key word: Transdermal delivery; skin; chemical penetration enhancers. 


\section{Introduction}

The transdermal route now ranks with oral treatment as the most successful innovative research area in drug delivey, with around $40 \%$ of the drug delivery candidate products under clinical evaluation related to transdermal or dermal system. The worldwide transdermal patch market approaches $£ 2$ billion, based on only ten drugs including scopolamine, nitroglycerine, clonidine, estrogen, testosterone, fentanyl, and nicotine, with a lidocaine patch soon to be marketed ${ }^{\prime}$. The success of a dermatological drug to be used for systemic drug delivery depends on the ability of the drug to penetrate through skin in sufficient quantities to achieve the desired therapeutic effect ${ }^{3}$. In this article, we review some uses of the more widely investigated chemical penetration enhancers and discuss their possible mechanisms of action.

Transdermal drug delivery is the administration of a therapeutic agent through intact skin for systemic effect. Transdermal drug delivery offers the following advantages over the oral route for controlled drug delivery ${ }^{3}$.

- Avoidance of hepatic first pass metabolism.

- Ability to discontinue administration by removal of the system.

- The ability to control drug delivery for a longer time than the usual gastrointestinal transit of oral dosage form.

- The ability to modify the properties of the biological barrier to absorption.

The method employed for modifying the barrier properties of the stratum corneum to enhance drug penetration and absorption through skin may be classified into the following categories ${ }^{4}$ :

1. Chemical enhancement

2. Physical enhancement

3. Biochemical enhancement
4. Supersaturation enhancement

5. Bioconvertable prodrug

\section{A brief review of skin structure}

The skin can be considered to have four distinct layers of tissue ${ }^{3}$.

1. Non-viable epidermis (stratum corneum)

2. Viable epidermis

3. Viable dermis

4. Subcutaneous connective tissue (hypodermis)

Non-viable epidermis (stratum corneum)

Stratum corneum is the outer most layer of skin, which is the actual physical barrier to most substance that come in contact with the skin. The stratum corneum is 10 to 20 cell layer thick over most of the body. Each cell is a flat, plate-like structure - 34-44 $\mu \mathrm{m}$ long, 25$36 \mu \mathrm{m}$ wide, 0.5 to $0.20 \mu \mathrm{m}$ thick - with a surface area of 750 to $1200 \mu \mathrm{m}^{2}$ stocked up to each other in brick like fashion. Stratum corneum consist of lipid (5-15\%) including phospholipids, glycosphingolipid, cholesterol sulfate and neutral lipid, protein (75-85\%) which is mainly keratin.

\section{Viable epidermis}

This layer of the skin resides between the stratum corneum and the dermis and has a thickness ranging from 50- $100 \mu \mathrm{m}$. The structure of the cells in the viable epidermis are physiochemically similar to other living tissues. Cells are held together by tonofibrils. The density of this region is not much different than water. The water content is about $90 \%$.

\section{Dermis}

Just beneath the viable epidermis is the dermis. It is a structural fibrin and very few cells are like it can be found histologically in normal tissue. Dermis thickness range from 2000 to $3000 \mu \mathrm{m}$ and consists of a matrix of loose connective tissue composed of fibrous 
protein embedded in an amphorphose ground substance.

\section{Subcutaneous connective tissue}

The subcutaneous tissue or hypodermis is not actually considered a true part of the structured connective tissue is composed of loose textured, white, fibrous connective tissue containing blood and lymph vessels, secretory pores of the sweat gland and cutaneous nerves. Most investigators consider drug permeating through the skin enter the circulatory system before reaching the hypodermis, although the fatty tissue could serve as a depot of the drug.

\section{Pathway of transdermal permeation}

Permeation can occur by diffusion via ${ }^{5}$ :

1. Transdermal permeation, through the stratum corneum.

2. Intercellular permeation, through the stratum corneum.

3. Transappendaged permeation, via the hair follicle, sebaceous and sweat glands.

Most molecules penetrate through skin via intercellular microroute and therefore many enhancing techniques aim to disrupt or bypass its elegant molecular architecture.

\section{Ideal characteristics of chemical penetration enhancers}

Ideally, penetration enhancers reversibly reduce the barrier resistance of the stratum corneum without damaging viable cells ${ }^{13,14}$. Some of the more desirable properties for penetration enhancers acting within the skin have been given as ${ }^{1}$ :

- They should be non-toxic, non-irritating and non-allergenic

- They would ideally work rapidly; the activity and duration of effect should be both predictable and reproducible.
- They should have no pharmacological activity within the body.

- The penetration enhancers should work unidirectionally, i.e., they should allow therapeutic agents into the body whilst preventing the loss of endogenous materials from the body.

- When removed from the skin, barrier properties should return both rapidly and fully to normal.

- They should be cosmetically acceptable with an appropriate skin feel.

Not surprisingly, no such material that possesses the above ideal properties has yet been discovered although some chemicals demonstrate several of the above attributes.

\section{Mechanism of chemical penetration enhancement}

Penetration enhancers may act by one or more of three main mechanisms ${ }^{1}$ :

1. Disruption of the highly ordered structure of stratum corneum lipid.

2. Interaction with intercellular protein.

3. Improved partition of the drug, coenhancer or solvent into the stratum corneum.

The enhancer act by altering one of three pathways. The key to altering the polar pathway is to cause protein conformational change or solvent swelling. The fatty acid enhancers increased the fluidity of the lipid protein portion of the stratum corneum. Some enhancers act on both polar and nonpolar pathway by altering the multilaminate pathway for penetration. Enhancers can increase the drug diffusivity through skin proteins. The type of enhancer employed has a significant impact on the design and development of the product $^{2}$. A useful way to consider factors affecting drug permeation rate through the stratum corneum is via the simple equation given below for steady state flux ${ }^{1}$. If we plot the cumulative mass of diffusant, $m$, passing per unit area through the membrane, at long 
time the graph approaches linearity and its slope its yield the steady flux,$d m / d t$

$$
d m / d t=D C_{o} K / h
$$

where $C_{o}$ is the constant concentration of drug in donor solution, $K$ is the partition coefficient of the solute between the membrane and the bathing solution, $D$ is the diffusion coefficient and $h$ is thickness of membrane.

From the above equation, we deduce the ideal properties of a molecule that would penetrating stratum corneum well. These are:

- Low molecular mass, preferably less than $600 \mathrm{Da}$, when $\mathrm{D}$ tends to be high.

- Adequate solubility in oil and water so that membrane concentration gradient may be high.

- High but balanced (optimal) K (if too large, may inhibit clearance by viable tissue)

- Low melting point, correlating with good solubility as predicted by ideal solubility theory.

\section{Chemical penetration enhancers}

Chemical substances temporarily diminishing the barrier of the skin and known as accelerants or sorption promoters can enhance drug flux. Several types are known

\section{Sulphoxides and similar chemicals}

Dimethyl sulphoxides (DMSO) is one of the earliest and most widely studied penetration enhancers. It is a powerful aportic solvent which hydrogen bonds with itself rather than with water. It is colourless, odourless and is hydroscopic and is often used in many areas of pharmaceutical sciences as a "universal solvent". DMSO alone has been applied topically to treat systemic inflammation. DMSO works rapidly as a penetration enhancer - spillage of the material onto the skin can be tasted in the mouth within a second. Although DMSO is an excellent accelerant, it does create problems. The effect of the enhancer is concentration-dependent and generally cosolvents containing > $60 \%$ DMSO are needed for optimum enhancement efficacy. However, at these relative high concentrations, DMSO can cause erythema and wheal of the stratum corneum. Denaturing of some skin proteins results in erythema, scaling, contact uticaria ,stinging and burning sensation ${ }^{6}$.

Since DMSO is problematic for use as a penetration enhancer, researchers have investigated a similar chemically-related material as a accelerant. DMAC and DMF are similarly powerful aportic solvents. However, Southwell and Barry, showing a 12-fold increase in the flux of caffeine permeating across a DMF-treated human skin, concluded that the enhancer caused irreversible membrane damage ${ }^{7}$. DMF irreversibly damages human skin membranes but has been found in vivo to promote the bioavailability of betamethasone-17-benzoate as measured by vasoconstrictor assay ${ }^{8,9}$. DMSO may also extract lipids, making the horny layer more permeable by forming aqueous channels ${ }^{12}$. The mechanism of the sulphoxide penetration enhancers is widely used to denature protein and, on application to human skin, has been shown to change the intercellular keratin conformation, from $\alpha$ helical to $B$ sheet $^{10,11}$.

\section{Azone}

Azone (1-dodecylazacycloheptan-2-one or laurocapran) was the first molecule specifically designed as a skin penetration enhancer. Azone is a colourless, odourless liquid with a melting point of $-7 \stackrel{\circ}{\circ} \mathrm{C}$ and it possesses a smooth, oily but yet non-greasy feel. Azone is a highly lipophilic material with a log p octanol / water of around 6.2 and it is soluble in and compatible with most organic solvents including alcohol and propylene glycol. Azone enhances the skin transport of a wide variety of drugs including steroids, antibiotics and antiviral agents. Azone is most effective at low concentrations, being employed typically between $0.1-5 \%$ but more 
often between $1-3 \%{ }^{13}$. Azone partitions into a bilayer lipid to disrupt their packing arrangement but integration into the lipid is unlikely to be homogeneous. Azone molecules may exist dispersed within the barrier lipoid or separate domains within the bilayer $^{14}$.

\section{Pyrrolidones}

Pyrrolidones have been used as permeation enhancers for numerous molecules including hydrophilic (e.g. mannitol and 5-flurouracil) and lipophilic (progesterone and hydrocortisone) permeants. N-methyl-2pyrolidone was employed with limited success as a penetration enhancer for captopril when formulated in a matrix-type transdermal patch $^{15}$. The pyrrolidones partition well into human stratum corneum within the tissue and they may act by altering the solvent nature of the membrane. Pyrrolidones have been used to generate reservoirs within the skin membrane. Such a reservoir effect offers a potential for sustained release of a permeant from the stratum corneum over extended time periods $^{16}$.

\section{Fatty acids}

Percutaneous drug absorption has been increased by a wide variety of long-chain fatty acids, the most popular of which is oleic acid. It is of interest to note that many penetration enhancers such as azone contain saturated or unsaturated hydrocarbon chains and some structure - activity relationships have been drawn from the extensive studies of Aungst who employed a range of fatty acids, acids, alcohols, sulphoxides, surfactants and amides as enhancers for naloxone ${ }^{17,18}$. Shin et $\mathrm{al}^{19}$ studied various penetration enhancers like glycols (diethylene glycol and tetraethylene glycol), fatty acids (lauric acid, myristic acid and capric acid) and nonic surfactant (polyoxyethylene-2-oleyl ether, polyoxy ethylene-2-stearly ether) on the release of triprolidone. Lauric acid in Propylene glycol enhanced the delivery of highly lipophilic antiestrogen ${ }^{20}$. Oleic acid greatly increased the flux of many drugs such as increasing the flux of salicylic acid 28-fold and 5-flurouracil flux 56-fold through human skin membrane in vitro $^{21}$. The enhancer interacts with and modifies the lipid domains of the stratum corneum as would be expected for a longchain fatty acid with cis- configuration ${ }^{13}$.

\section{Essential oil, terpenes and terpenoids}

Terpenes are found in essential oils, and are compounds comprising of only carbon, hydrogen and oxygen atoms, but which are not aromatic. Numerous terpenes have long been used as medicines as well as flavoring and fragrance agents. The essential oils of eucalyptus, chenopodium and ylang-ylang have been found to be effective penetration enhancers for 5-flouorouracil transversing human skin in vivo ${ }^{22}$. Cornwell et $\mathrm{al}^{23}$ investigated the effect of 12 sesquiterpenes on the permeation of 5-flurouracil in human skin. Pretreatment of epidermal membranes with sesquiterpene oil or using solid sesquiterpenes saturated in dimethyl isosorbide increased the absorption of 5flurouracil. L-menthol has been used to facilitate in vitro permeation of morphine hydrochloride through hairless rat $\operatorname{skin}^{24}$ as well as diffusion of imipramine hydrochloride across rat skin and hydrocortisone through hairless mouse skin ${ }^{25,26}$.

One mechanism by which this agent operates is to modify the solvent nature of the stratum corneum, thus improving drug partitioning into the tissue. Many terpenes permeate human skin well and large amounts of terpene have been found in the epidermis after application from a matrix-type patch. Terpenes may also modify drug diffusivity through the membrane. During steady state permeation experiments using terpenes as penetration enhancers, the lag time for permeation was usually reduced, indicating some increase in drug diffusivity 
through the membrane following terpene treatment ${ }^{13}$.

\section{Oxazolidinones}

Oxazolidinones are a new class of chemical agents which have the potential for use in many cosmetic and personal care product formulations. This is due to their ability to localize co-administered drug in skin layers, resulting in low systemic permeation ${ }^{27,28}$. The structural features of these permeation enhancers are closely related to sphingosine and ceramide lipids which are naturally found in the upper skin layers. Oxazolidinones such as 4-decyloxazolidin-2-one has been reported to localize the delivery of many active ingredients such as retinoic acid and diclofenac sodium in skin layers ${ }^{29}$. This compound has a higher molecular weight and lipophilicity than other solvent-type enhancers, physical characteristics that may be beneficial in terms of a reduction in local toxicity because of the lack of effective absorption of these enhancers into the lower skin layers where irritation is likely to be occur.

\section{Urea}

Urea promotes transdermal permeation by facilitating hydration of the stratum corneum and by the formation of hydrophilic diffusion channels within the barrier. Cyclic urea permeation enhancers are biodegradable and non-toxic molecules consisting of a polar parent moiety and a long chain alkyl ester group. As a result, enhancement mechanism may be a consequence of both hydrophilic activity and lipid disruption mechanism ${ }^{4}$.

\section{Conclusion}

Skin permeation enhancement technology is a rapidly developing field which would significantly increase the number of drugs suitable for transdermal drug delivery, with the result that skin will become one of major routes of drug administration in the next decade. Research in this area has proved the usefulness of chemical penetration enhancers in the enhancement of drug permeation through skin. The chemical penetration enhancement methods discussed in this review are promising. Focus should be on skin irritation with a view to selecting penetration enhancers which possess optimum enhancement effects with minimal skin irritation.

\section{Acknowledgements}

The authors express their sincere thanks to Secretary Sri. Prashant Gadhak and Principal V.K.Dhesmukh, M.E.S College of Pharmacy, Sonai for providing the required facilities.

\section{References}

1. Barry BW, Dermatological formulation: percutaneous absorption. Marcel Dekker, New York, 1983.

2. Barry BW, William AC. In: Swarbrick J (ed), Boylon JC. Encyclopedia of pharmaceutical technology $\mathrm{Vol}$ II, Marcel Dekker: Inc new York 1995, pp 4993.

3. Kanikkannan N, Kandimalla K, Lamba SS, Singh M. Structures activity relationship of chemical penetration enhancers in transdermal drug delivery. Current Medicinal Chemistry 1999; 6: 593-608.

4. Singh PB, Choudhury PK. Penetration enhancers for transdermal drug delivery of systemic agents. $J$ PharmRes 2007; 6: 44-50.

5. Cleary GW. In: Lange RS, Wise DL (eds). Medical application of controlled release, CRC Press, Boca Raton, Florida, 1984, Vol I, pp 203-45.

6. Kligman AM. Topical pharmacology and toxicology of dimethylsulfoxide. J Am Med Assoc. 1965; 193: 796-804.

7. Southwell D, Barry BW. Penetration enhancers for human skin: mode of action of 2-Pyrrolidone and dimethylformamide on partition and diffusion of model compound water, $n$-alcohol and caffeine. J Invest Dermatol 1984; 82 :507515.

8. Barry BW, Southwell D, Woodford R. Optimization of bioavailability of topical steroid: penetration enhancers under occulsion. J Invest Dermatol 1984; 82: 49-52.

9. Bennett SL, Barry BW, Woodford R. Optimization of bioavailability of topical steroid: non -occluded penetration enhancers under thermodynamic control. J Pharm Pharmacol 1984; 37: 294304.

10. Oerta RP.I. Protein conformational change induced in human stratum corneum by organic sulphoxides: an infrared spectroscopic investigation. Biopolymer 1997; 16: 2329-2345. 
11. Anigbog ANC, William AC, Barry BW, Edwards HGM. Fourier transform raman spectroscopy of interaction between the penetration enhancer dimethylsulphoxide and human stratum corneum.Int J Pharm 1995; 125: 265-282.

12. Menczel EM. Delipidization of the cutaneous permeability barrier and Percutaneous penetration. In: Smith EW, Maibach HI.(eds)Percutaneous penetration enhancers, CRC Press, Boca Raton FL, pp 383-392.

13. William AC, Barry BW. Penetration enhancer. Adv Drug Deliv 2004; 56:603-618.

14. Hoogstrate AJ, Verhoef J, Brusee, ljzerman AP, Spies $F$, Bodde HE. Kinetic, ultrastructural aspects and molecular modeling of transdermal peptide flux enhancement by $\mathrm{N}$-alkylazocylohepton. Int J Pharm 1991; 76: 37-47.

15. Park ES, Chang SJ, Rhee YS, Chis C. Effect of adhesive and permeation enhancer on the skin permeation of captopril. Drug Deve Ind Pharmacy 2001; 27: 975-980.

16. Jungbauer FHW, Coenraods PJ, Kardaun SH. Toxic hygroscopic contact reaction to $\mathrm{N}$-methyl-2Pyrrolidone. Contact Dermatitis 2001; 45: 303304.

17. Aungst BJ, Rogers NJ, Shefter E. Enhancement of naloxone penetration through human skin in vitro using fatty acid, fatty alcohol, surfactant, sulfoxide and amide. Int J Pharm 1986; 33: 244-247.

18. Aungst BJ. Structure effect studies of fatty acid isomers as skin penetration enhancers and skin irritant. Pharm Res 1989; 6:244-247.

19. Shin SC, Lee HJ. Controlled release of triprolidone using ethylene-vinyl acetate membrane and matrix system. Int J Pharm 2002; 54: 201-206.

20. Funke AP, Schiller R, Motzkus HW, Gunther C, Muller $R H$, Lipp R. Transdermal delivery of highly lipophilic drug: in vitro fluxes of antiestrogen permeation enhancers, and solvents from liquid formation. Pharm Res 2002; 19: 661-668.

21. Goodman M, Barry BW. Lipid-protein partitioning theory of skin enhancer activity. Finit dose technique. Int J Pharm 1989; 57: 29-40.

22. William AC, Barry BW. Essential oil as novel human skin penetration enhancer. Int J Pharm 1989; 57: R7-R9.

23. Cornwell PA, Barry BW. Sesquiterpene components of volatile oils as skin penetration enhancers for the hydrophilic permeant 5-fluorouracil. J Pharm Pharmacol 1994; 46: 261-269.

24. Morimoto $H$, Woda $Y$, Seki T, Sugibayashi $K$. In vitro skin permeation of morphin hydrochloride during the finite application of penetrationenhancing system containing water, ethanol and L-menthol. Biol Pharm Bull 2002; 25: 134136.

25. Jain AK, Thomas NS, Panchagnula R. Transdermal drug delivery of imipramine hydrochloride. I. Effect of terpenes. J Control Rel 2002; 79: 93101.

26. EL-Kuttan AF, Asbill CS, Michniak BB. The effect of terpenes enhancer lipophilicity on the percutaneous permeation of hydrocortisone formulated in HPMC gel system. Int J Pharm 2000; 198: 179-189.

27. Rajadhyaksha V, Pfister WR. Oxazolidinones. Drug Cosmet Ind 1996; 1: 36-47.

28. Seth $B$. Transdermal delivery using decycyloxazolidin2-one.Arzeim-forsch. Drug Res1999; 42: 120122.

29. Asbill CS, Michniak BB. Percutaneous penetration enhancers: Local versus transdermal activity. Research focus 2000; 3: 36-41. 